\title{
Síntesis y caracterización de electrocatalizadores basados en calcogenuros nanoestructurados de $\mathrm{Ru}-\mathrm{WS}_{2} / \mathrm{C}$ y Ru-WS$/$ NTC
}

\author{
Asunción Arango ${ }^{a}$, A. Fernández-Madrigal ${ }^{\mathrm{b}}$, Bonfilio Arango ${ }^{\mathrm{a}}$, Manuel Sánchez Rubio ${ }^{\mathrm{c}}$ y Y. Gochi- \\ Ponce $^{\mathrm{c} *}$ \\ ${ }^{a}$ División de Estudios de Posgrado e Investigación, Instituto Tecnológico de Oaxaca, Ave. Ing. Víctor Bravo Ahuja esq. Calz. \\ Tecnológico 125, Oaxaca, Oax., México. \\ ${ }^{b}$ Centro de Investigación en Energía-UNAM, Privada Xochicalco S/N. C.P. 62580 Temixco, Morelos, México \\ ${ }^{c}$ Departamento de Ingeniería Química u Bioquímica, Instituto Tecnológico de Oaxaca, Ave. Ing. Víctor Bravo Ahuja esq. Calz. \\ Tecnológico 125, Oaxaca, Oax., México. \\ *E-mail: yadira.gochi@gmail.com
}

Recibido 10 de enero de 2013, Aceptado 1 de febrero de 2013

\section{Resumen}

En este trabajo se llevó a cabo una reacción química entre metales de transición, específicamente tiosales de tungsteno y rutenio para sintetizar dos precursores catalíticos soportados en carbón Vulcan y un precursor catalítico soportado en nanotubos de carbono (NTC). Los materiales se caracterizaron por difracción de rayos X (DRX), microscopía electrónica de barrido (MEB) y microscopía electrónica de transmisión (MET). El electrocatalizador comercial de Pt/C al $20 \%$ en peso metálico se utilizó para comparar el desempeño catódico de los electrocatalizadores sintetizados y basados en calcogenuros de $\mathrm{Ru}-\mathrm{WS}_{2}$. Los electrocatalizadores obtenidos se analizaron mediante técnicas voltamperométricas para la reacción de reducción de oxígeno (RRO) en medio ácido. El electrocatalizador $\mathrm{Ru}_{\mathrm{X}} \mathrm{W}_{\mathrm{Y}} \mathrm{S}_{\mathrm{Z}} / \mathrm{C}$ resultó $38 \%$ más eficiente para la generación de corriente como cátodo respecto al electrocatalizador $\mathrm{Ru}_{\mathrm{X}} \mathrm{W}_{\mathrm{Y}} \mathrm{S}_{2 Z} / \mathrm{C}$. Este resultado se atribuye a la alta dispersión y homogeneidad en el tamaño nanométrico de las partículas metálicas de la fase activa.

Palabras clave: electrocatalizadores, calcogenuros, nanoestructurados, celdas de combustible.

\section{Introducción}

Debido a la necesidad que existe actualmente de reducir el nivel de contaminantes presentes en la atmósfera, principales causantes de problemas que afectan seriamente a la humanidad como el efecto invernadero y la variación de los regímenes climatológicos, surge la necesidad de buscar nuevas soluciones que contribuyan al mejoramiento de estos problemas. Una buena solución es cambiar de base energética utilizando un combustible con el cual se puedan eliminar o reducir notablemente las emisiones de contaminantes a la atmósfera.

Las celdas de combustible de intercambio protónico (PEM) representan una alternativa energética, son dispositivos que convierten en forma directa la energía química de los reactantes en energía eléctrica. Uno de los elementos esenciales para el buen funcionamiento de las celdas de combustible tipo PEM es el electrocatalizador, que normalmente es de un material caro como el platino. Este material se encuentra en el electrodo y presenta características particulares como: porosidad, alta área electroquímicamente activa, inerte y buen conductor [1].

La reacción global en una celda de combustible tipo PEM está controlada por la reacción catódica, por ser ésta cercana a cinco órdenes de magnitud más lenta que la reacción anódica. La electrocatálisis de la reacción de reducción de oxígeno es de interés práctico, teórico y su importancia electroquímica es esencial para comprender los procesos fisicoquímicos que permitirán mejorar el diseño de electrocatalizadores más eficientes. Los electrocatalizadores nanométricos han mostrado un alto desempeño para la reducción de oxígeno (RRO) y esta alta actividad depende no sólo del tamaño de las partículas, sino de la naturaleza del soporte y de los métodos de preparación. La síntesis y caracterización de nuevos materiales con actividad catalítica y selectividad alta para la RRO molecular, han merecido un trabajo extensivo en las últimas décadas [2], debido a los elevados costos de los materiales con base en platino.

Hoy en día la búsqueda de materiales alternos al platino comercial, que es el electrocatalizador más eficiente para las celdas tipo PEM, representa uno de los principales desafíos en el campo de la electrocatálisis, ya que hasta hoy, no se ha encontrado un electrocatalizador que reúna todas las bondades que éste presenta en medio ácido y que pueda ser utilizado como electrodo catódico o anódico y que sea a su vez, menos costoso [3-4].

Por lo tanto, en esta investigación se proponen 
nanoelectrocatalizadores basados en calcogenuros ternarios. En la obtención de los electrocatalizadores propuestos se partirá de nanoprecursores de RuxWySz los cuales servirán como electrodos catódicos que serán más eficientes y económicos que el platino comercial.

\section{Parte experimental}

Se sintetizaron dos nanoprecursores $\mathrm{Ru}_{\mathrm{X}} \mathrm{W}_{\mathrm{Y}} \mathrm{S}_{\mathrm{Z}} \mathrm{y}$ $\mathrm{Ru}_{\mathrm{X}} \mathrm{W}_{\mathrm{Y}} \mathrm{S}_{2 Z}$, los cuales fueron preparados por reacción química utilizando tiosales de tungsteno $\left(\left(\mathrm{NH}_{4}\right)_{2} \mathrm{WS}_{4}\right)$ y una sal de hexaclororutenato $\left(\mathrm{NH}_{4}\right)_{2} \mathrm{RuCl}_{6}$ (Sigma-Aldrich) éstas se sintetizaron previamente por un método empleado en la preparación de catalizadores para HDS [5].

A continuación se presentan las reacciones químicas propuestas cuya relación molar es 1:1 en la ecuación 1 y la relación molar es 1:2 en la ecuación 2.

$\left(\mathrm{NH}_{4}\right)_{2} \mathrm{RuCl}_{6}+\left(\mathrm{NH}_{4}\right)_{2} \mathrm{WS}_{4} \rightarrow\left(\mathrm{NH}_{4}\right)_{4} \mathrm{RuWS}_{4}+3 \mathrm{Cl}_{2}(1)$

$\left(\mathrm{NH}_{4}\right)_{2} \mathrm{RuCl}_{6}+2\left(\mathrm{NH}_{4}\right)_{2} \mathrm{~W} \rightarrow\left(\mathrm{NH}_{4}\right)_{6} \mathrm{RuW}_{2} \mathrm{~S}_{8}+3 \mathrm{Cl}_{2}$

Las sales metálicas se agitaron constantemente a temperatura ambiente. Las soluciones resultantes se mezclaron con el soporte catalítico, que fue carbón Vulcan (E-TEK) o nanotubos de carbón NTC (el tipo de NTC fue utilizado tal como se recibió sin tratamientos previos), es decir, $20 \%$ en peso de la fase activa y $80 \%$ soporte, se mantuvieron en agitación constante. Los precipitados se filtraron, se lavaron con agua destilada y se secaron a temperatura ambiente. Los nanoprecursores obtenidos en la síntesis se nombran como: $\mathrm{Ru}_{\mathrm{X}} \mathrm{W}_{\mathrm{Y}} \mathrm{S}_{\mathrm{Z}} / \mathrm{C}, \mathrm{Ru}_{\mathrm{X}} \mathrm{W}_{\mathrm{Y}} \mathrm{S}_{2 Z} / \mathrm{C}$ y $\mathrm{Ru}_{\mathrm{X}} \mathrm{W}_{\mathrm{Y}} \mathrm{S}_{2 Z} / \mathrm{NTC}$.

La caracterización fisicoquímica de los nanoprecursores obtenidos, se llevó a cabo por la técnica de análisis termogravimétrico (TGA), lo cual permitió conocer el comportamiento de la masa de la muestra analizada en función de la temperatura. Los nanoprecursores soportados fueron tratados térmicamente a una temperatura de $300{ }^{\circ} \mathrm{C}$ bajo una atmósfera de $\mathrm{N}_{2} / \mathrm{H}_{2}(75 \% \mathrm{~V} / \mathrm{V})$ por $2 \mathrm{~h}$ para obtener los electrocatalizadores. Además, los nanoprecursores tratados térmicamente se caracterizaron por DRX donde las mediciones se hicieron con una radiación de $\mathrm{Cu}-\mathrm{K} \alpha$ de un difractómetro Rigaku modelo DMAX 2200, las técnicas de MEB en un microscopio electrónico de barrido marca LEO VP que operó a $10 \mathrm{Kv}$ y por MET en un microscopio electrónico de transmisión de emisión de campo Jeol modelo JEM-2200FS operado a $200 \mathrm{kV}$, lo cual permitió conocer las estructuras cristalinas y morfología de cada uno de estos.

Posteriormente se prepararon dos tintas:

La primera por dispersión acuosa de los catalizadores $\quad \mathrm{Ru}_{\mathrm{X}} \mathrm{W}_{\mathrm{Y}} \mathrm{S}_{\mathrm{Z}} / \mathrm{C}, \quad \mathrm{Ru}_{\mathrm{X}} \mathrm{W}_{\mathrm{Y}} \mathrm{S}_{2 \mathrm{Z}} / \mathrm{C} \quad$ y $\mathrm{Ru}_{\mathrm{X}} \mathrm{W}_{\mathrm{Y}} \mathrm{S}_{2 Z} / \mathrm{NTC}$ con una relación $5 \mathrm{~g} / \mathrm{L}$.

La segunda es una mezcla $(50: 50 \% \mathrm{~V} / \mathrm{V})$ de nafion y agua desionizada, respectivamente.

Estas tintas fueron depositadas en carbón vítreo de $3 \mathrm{~mm}$ de diámetro y secadas bajo un flujo de nitrógeno para realizar las pruebas electroquímicas usando voltamperometría cíclica (VC) y lineal (VL). El equipo utilizado fue en un potenciostato EG\&G Princeton Applied Research Model 263A controlado por una computadora personal, un radiómetro y un electrodo de disco rotatorio (EDR). El experimento fue llevado a cabo a temperatura ambiente en un flujo de gas nitrógeno para purgar el oxígeno dentro de una solución de $0.5 \mathrm{M} \mathrm{H}_{2} \mathrm{SO}_{4}$ en una celda de tres electrodos para la voltamperometría cíclica y un flujo de oxígeno fue utilizado hasta saturar la solución para la voltamperometría lineal.

\section{Resultados y discusión}

La Figura 1 presenta los difractogramas de rayos $\mathrm{X}$ de los catalizadores $\mathrm{Ru}_{\mathrm{X}} \mathrm{W}_{\mathrm{Y}} \mathrm{S}_{\mathrm{Z}} / \mathrm{C}, \mathrm{Ru}_{\mathrm{X}} \mathrm{W}_{\mathrm{Y}} \mathrm{S}_{2 \mathrm{Z}} / \mathrm{C}$ y $\mathrm{Ru}_{\mathrm{X}} \mathrm{W}_{\mathrm{Y}} \mathrm{S}_{2 Z} / \mathrm{NTC}$. Se puede observar que en los tres catalizadores el comportamiento es el mismo, un pico prominente a $24^{\circ}$ (2theta) el cual es debido a la presencia de la fase cristalina del carbón Vulcan y Nanotubos de carbón, respectivamente. La ausencia de algunas señales del $\mathrm{WS}_{2}$ se atribuye a que se generan nanopartículas altamente exfoliadas, ya que estos materiales presentan estructuras hexagonales de capas S-W-S [6], cuyas reflexiones se podrían confirmar con otras técnicas de caracterización complementarias. Sin embargo, aparece una señal a $45^{\circ}$ ( 2 theta), que corresponde a la dirección cristalina (103) del $\mathrm{WS}_{2}$, coincide con la dirección (111) del carbón Vulcan y las direcciones cristalinas (100) (002) y (101) que se identificaron a 42.21, 44.08 y 78.48 (2theta) del Ru, que están reportadas en las tablas JCPDS 6-663. Por lo tanto, debido a que algunas de las fases cristalinas del carbón Vulcan, de los NTC y el Ru también se traslapan, se realizaron microanálisis por EDS para corroborar la presencia de las mismas. 


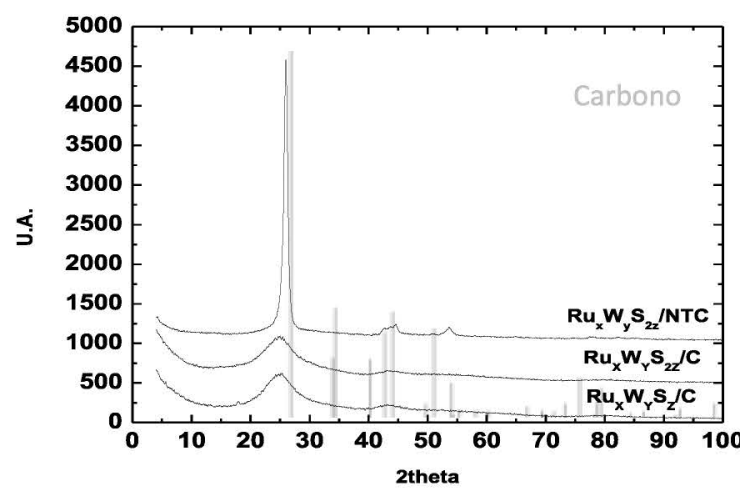

Figura 1. Difractogramas de los catalizadores obtenidos

Las imágenes por MEB de los catalizadores $\mathrm{Ru}_{\mathrm{X}} \mathrm{W}_{\mathrm{Y}} \mathrm{S}_{\mathrm{Z}} / \mathrm{C}, \quad \mathrm{Ru}_{\mathrm{X}} \mathrm{W}_{\mathrm{Y}} \mathrm{S}_{2 \mathrm{Z}} / \mathrm{C}$ y $\quad \mathrm{Ru}_{\mathrm{X}} \mathrm{W}_{\mathrm{Y}} \mathrm{S}_{2 \mathrm{Z}} / \mathrm{NTC}$ aparecen en la Figura 2 mediante las micrografías obtenidas. En la Figura 2a) se presenta el catalizador $\mathrm{Ru}_{\mathrm{X}} \mathrm{W}_{\mathrm{Y}} \mathrm{S}_{\mathrm{Z}} / \mathrm{C}$, se observa un gran número de partículas de tamaño pequeño que están definidas por un aspecto poroso tipo esponja, la Figura 2b) presenta partículas irregulares del catalizador $\mathrm{Ru}_{\mathrm{X}} \mathrm{W}_{\mathrm{Y}} \mathrm{S}_{2 Z} / \mathrm{C}$, se distinguen cúmulos de mayor tamaño en comparación con el catalizador $\mathrm{Ru}_{\mathrm{X}} \mathrm{W}_{\mathrm{Y}} \mathrm{S}_{\mathrm{Z}} / \mathrm{C}$ y en la Figura 2c) se aprecian algunas partículas brillantes y aglomeradas del $\mathrm{Ru}_{\mathrm{X}} \mathrm{W}_{\mathrm{Y}} \mathrm{S}_{2 Z} / \mathrm{NTC}$, esto es debido al tipo de depósito de la fase metálica y su interacción con los nanotubos de carbón multipared, se observa una distribución aleatoria de los NTC y diferentes morfologías. El catalizador $\mathrm{Ru}_{\mathrm{X}} \mathrm{W}_{\mathrm{Y}} \mathrm{S}_{\mathrm{Z}} / \mathrm{C}$ es más homogéneo que el $\mathrm{Ru}_{\mathrm{X}} \mathrm{W}_{\mathrm{Y}} \mathrm{S}_{2 Z} / \mathrm{C}$, la formación de cúmulos se atribuye a que un incremento en la relación molar de la tiosal de tungsteno en la síntesis, no favorece la dispersión de la fase activa y por lo tanto, el catalizador $\mathrm{Ru}_{\mathrm{X}} \mathrm{W}_{\mathrm{Y}} \mathrm{S}_{2 \mathrm{Z}} / \mathrm{C}$ presenta partículas más grandes. Este resultado también es evidente en el catalizador $\mathrm{Ru}_{\mathrm{X}} \mathrm{W}_{\mathrm{Y}} \mathrm{S}_{2 \mathrm{Z}} / \mathrm{NTC}$. Por otra parte, se asume que el azufre se absorbe fácilmente en el carbón, sin embargo, el mecanismo e interacción de estos elementos durante la reacción dependerá, en gran medida, de la estructura y el tipo de arreglo atómico de las redes hexagonales de los soportes (Vulcan y NTC), por lo tanto, uno de los efectos que se presentan en este estudio es que una aglomeración de la fase activa, no permite tener una buena dispersión del Ru y W.

Los microanálisis de los catalizadores obtenidos por EDS muestran una relación atómica para: $\mathrm{Ru}_{\mathrm{X}} \mathrm{W}_{\mathrm{Y}} \mathrm{S}_{\mathrm{Z}} / \mathrm{C}$ de $(\mathrm{X}=1, \mathrm{Y}=1$ y $\mathrm{Z}=2), \mathrm{Ru}_{\mathrm{X}} \mathrm{W}_{\mathrm{Y}} \mathrm{S}_{2 Z} / \mathrm{Cde}$ $(X=1, Y=1$ y $Z=3)$ y $R u_{X} W_{Y} S_{2 Z} /$ NTC de $(X=1$, $\mathrm{Y}=1$ y $\mathrm{Z}=2$ ); estas relaciones atómicas son obtenidas después de realizar los tratamientos térmicos.

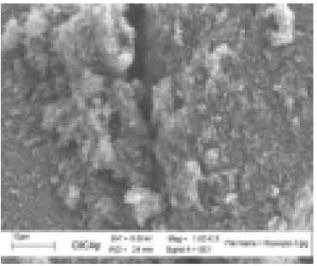

a) $\mathrm{Ru}_{\mathrm{X}} \mathrm{W}_{\mathrm{Y}} \mathrm{S}_{\mathrm{Z}} / \mathrm{C}$

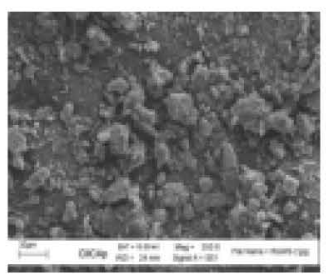

b) $\mathrm{Ru}_{\mathrm{X}} \mathrm{W}_{\mathrm{Y}} \mathrm{S}_{2 Z} / \mathrm{C}$

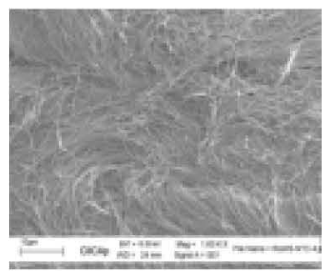

c) $\mathrm{Ru}_{\mathrm{X}} \mathrm{W}_{\mathrm{Y}} \mathrm{S}_{2 \mathrm{Z}} / \mathrm{NTC}$

Figura 2. Imágenes de MEB a) $\mathrm{Ru}_{\mathrm{X}} \mathrm{W}_{\mathrm{Y}} \mathrm{S}_{\mathrm{Z}} / \mathrm{C}$, b) $\mathrm{Ru}_{\mathrm{X}} \mathrm{W}_{\mathrm{Y}} \mathrm{S}_{2 Z} / \mathrm{C}$ y c) $\mathrm{Ru}_{\mathrm{X}} \mathrm{W}_{\mathrm{Y}} \mathrm{S}_{2 \mathrm{Z}} / \mathrm{NTC}$

La micrografía del catalizador de $\mathrm{Ru}_{\mathrm{X}} \mathrm{W}_{\mathrm{Y}} \mathrm{S}_{\mathrm{Z}} / \mathrm{C}$ obtenida por TEM se presenta en la Figura 3 a), se observan nanopartículas de $\mathrm{Ru}$ y $\mathrm{WS}_{2}$ que están altamente distribuidas sobre el soporte (carbón Vulcan), el tamaño promedio de las nanopartículas de $\mathrm{Ru}$ es de $2 \mathrm{~nm}$. Se realizó un análisis para determinar la distancia interatómica de los planos de $\mathrm{WS}_{2}$, debido a que en la literatura se reporta un valor de distancia interplanar de $0.6 \mathrm{~nm}[9,10]$ para esta fase cristalina, el valor reportado coincide con los resultados obtenidos del análisis de varias zonas de la muestra. Además, se confirman los análisis discutidos previamente por XRD y MEB que sugieren que el catalizador sintetizado $\mathrm{Ru}_{\mathrm{X}} \mathrm{W}_{\mathrm{Y}} \mathrm{S}_{\mathrm{Z}} / \mathrm{C}$ está basado en nanoestructuras exfoliadas de $\mathrm{WS}_{2}$ sobre la superficie de las nanopartículas de rutenio y que están altamente dispersas en el soporte de carbón.

En la imagen de la Figura 3 b) aparece la micrografia del catalizador $\mathrm{Ru}_{\mathrm{X}} \mathrm{W}_{\mathrm{Y}} \mathrm{S}_{2 Z} / \mathrm{C}$ por MET, la cual corrobora los resultados obtenidos por MEB, que mostraron que la fase metálica forma aglomerados y no se dispersa uniformemente en el soporte (carbón Vulcan).

En la imagen de la Figura 3 c) se observa la morfología del catalizador $\mathrm{Ru}_{\mathrm{X}} \mathrm{W}_{\mathrm{Y}} \mathrm{S}_{2 \mathrm{Z}} / \mathrm{NTC}$ obtenida por MET, se aprecia que el tipo de depósito de las partículas metálicas no fue uniforme en los NTC y se determinó un tamaño promedio de partícula de $10 \mathrm{~nm}$. Además, en esta misma imagen pueden apreciarse dos tipos de morfologías diferentes de los NTC. 


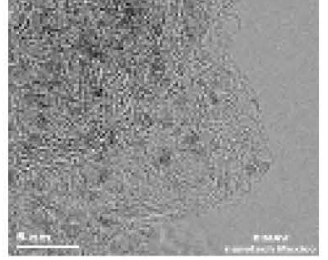

a) $\mathrm{Ru}_{\mathrm{X}} \mathrm{W}_{\mathrm{Y}} \mathrm{S}_{\mathrm{Z}} / \mathrm{C}$

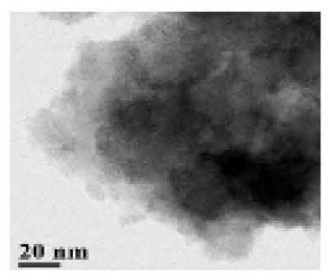

b) $\mathrm{Ru}_{\mathrm{X}} \mathrm{W}_{\mathrm{Y}} \mathrm{S}_{2 Z} / \mathrm{C}$

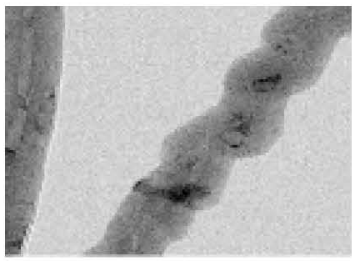

c) $\mathrm{Ru}_{\mathrm{X}} \mathrm{W}_{\mathrm{Y}} \mathrm{S}_{2 Z} / \mathrm{NTC}$

Figura 3. Imágenes de $M E T$ a) $\mathrm{Ru}_{\mathrm{X}} \mathrm{W}_{\mathrm{Y}} \mathrm{S}_{\mathrm{Z}} / \mathrm{C}, \mathrm{b}$ ) $\mathrm{Ru}_{\mathrm{X}} \mathrm{W}_{\mathrm{Y}} \mathrm{S}_{2 \mathrm{Z}} / \mathrm{C}$ y c) $\mathrm{Ru}_{\mathrm{X}} \mathrm{W}_{\mathrm{Y}} \mathrm{S}_{2 Z} / \mathrm{NTC}$

La Figura 4 a) presenta una micrografía obtenida por TEM de las nanopartículas del catalizador $\mathrm{Ru}_{\mathrm{X}} \mathrm{W}_{\mathrm{Y}} \mathrm{S}_{\mathrm{Z}} / \mathrm{C}$ obtenida con electrones dispersados a altos ángulos. Se midieron 27 nanopartículas en diferentes zonas de la muestra para generar un histograma de distribución que aparece en la Figura 4 b). Los resultados corroboran que el tamaño de las nanopartículas de la fase metálica en promedio es de 2 t/- $0.6 \mathrm{~nm}$, como se mencionó anteriormente en el análisis por MET. Este resultado es importante ya que estudios previos han demostrado que a menor tamaño de partícula, aumenta la actividad electrocatalítica [7,8].
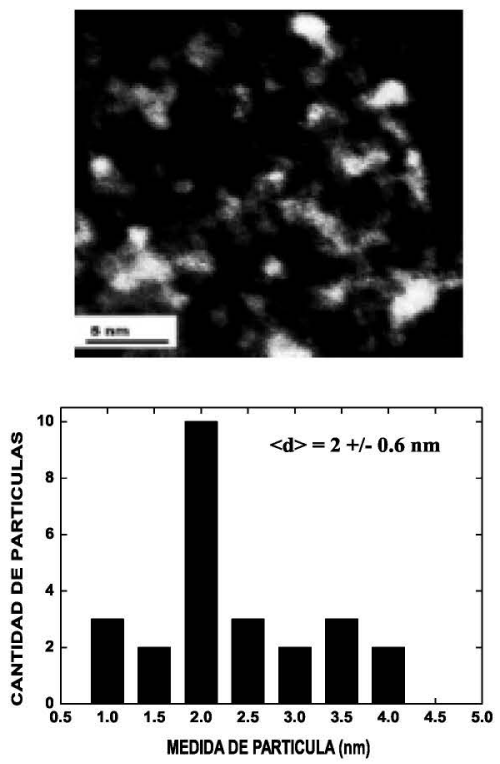

Figura 4. a) Imagen por TEM de RuxWySz/C y b) Histograma de la distribución del tamaño de partícula de $\mathrm{RuxWySz/C}$.
La Figura 5 muestra los voltamperogramas obtenidos de los electrocatalizadores $\mathrm{Ru}_{\mathrm{X}} \mathrm{W}_{\mathrm{Y}} \mathrm{S}_{\mathrm{Z}} / \mathrm{C}$, $\mathrm{Ru}_{\mathrm{X}} \mathrm{W}_{\mathrm{Y}} \mathrm{S}_{2 Z} / \mathrm{C}$ y $\mathrm{Ru}_{\mathrm{X}} \mathrm{W}_{\mathrm{Y}} \mathrm{S}_{2 Z} / \mathrm{NTC}$. En la VC del $\mathrm{Ru}_{\mathrm{X}} \mathrm{W}_{\mathrm{Y}} \mathrm{S}_{\mathrm{Z}} / \mathrm{C}$, la región anódica de adsorción $\mathrm{y}$ desorción del hidrógeno (0.05 V-0.2 V vs RHE) indica que el $\mathrm{WS}_{2}$ modifica significativamente la superficie del rutenio [11, 12]; del mismo modo, se observa que la corriente de oxidación causada por la descomposición de las especies relacionadas con el agua en el rutenio empieza a $0.3 \mathrm{~V}$ y tiene un fuerte aumento de corriente a partir de $0.8 \mathrm{~V}$, lo que se ha reportado anteriormente [13] como la oxidación de $\mathrm{S}$ dentro de la reacción redox: $\mathrm{H}_{2} \mathrm{SO}_{4}$ $+6 \mathrm{H}^{+}+6 \mathrm{e}^{-}=\mathrm{S}+4 \mathrm{H}_{2} \mathrm{O}$. Además el pico de reducción se observa a $0.4 \mathrm{~V}$ debido a la reducción de los óxidos de rutenio. Por otra parte, en la Figura 5 se muestran las VC de los electrocatalizadores $\mathrm{Ru}_{\mathrm{X}} \mathrm{W}_{\mathrm{Y}} \mathrm{S}_{2 Z} / \mathrm{C}$ y $\mathrm{Ru}_{\mathrm{X}} \mathrm{W}_{\mathrm{Y}} \mathrm{S}_{2 Z} / \mathrm{NTC}$, en donde la adsorción y la desorción del hidrógeno presentan un comportamiento similar al $\mathrm{Ru}_{\mathrm{X}} \mathrm{W}_{\mathrm{Y}} \mathrm{S}_{\mathrm{Z}} / \mathrm{C}$ discutido anteriormente. Sin embargo, la densidad de corriente es menor para el $\mathrm{Ru}_{\mathrm{X}} \mathrm{W}_{\mathrm{Y}} \mathrm{S}_{2 Z} / \mathrm{NTC}$ y mayor para el $\mathrm{Ru}_{\mathrm{X}} \mathrm{W}_{\mathrm{Y}} \mathrm{S}_{\mathrm{Z}} /$ C. En general, la corriente de oxidación empieza a $0.3 \mathrm{~V}$ con un aumento considerable a partir de aproximadamente $0.8 \mathrm{~V}$ por la oxidación del S. Finalmente, se observa un pico muy pequeño a $0.45 \mathrm{~V}$ relacionado con la reducción de óxidos de rutenio, esta señal es ligeramente más notable en los electrocatalizadores de relación molar 1:2.

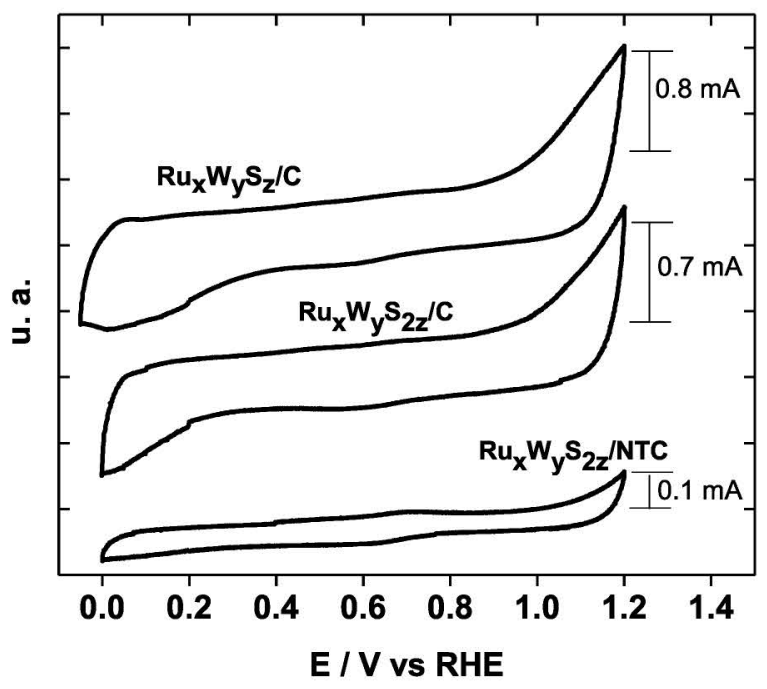

Figura 5. Voltamperogramas cíclicos de $\mathrm{Ru}_{\mathrm{X}} \mathrm{W}_{\mathrm{Y}} \mathrm{S}_{\mathrm{Z}} / \mathrm{C}$ $\mathrm{Ru}_{\mathrm{X}} \mathrm{W}_{\mathrm{Y}} \mathrm{S}_{2 Z} / \mathrm{C}$ y $\mathrm{Ru}_{\mathrm{X}} \mathrm{W}_{\mathrm{Y}} \mathrm{S}_{2 Z} / \mathrm{NTC}$. 
En la Figura 6 se presenta una comparación entre las VLs de los materiales sintetizados $\mathrm{Ru}_{\mathrm{X}} \mathrm{W}_{\mathrm{Y}} \mathrm{S}_{\mathrm{Z}} / \mathrm{C}$ $\mathrm{Ru}_{\mathrm{X}} \mathrm{W}_{\mathrm{Y}} \mathrm{S}_{2 Z} / \mathrm{C}$ y $\mathrm{Ru}_{\mathrm{X}} \mathrm{W}_{\mathrm{Y}} \mathrm{S}_{2 Z} / \mathrm{NTC}$, con respecto al $\mathrm{Pt} / \mathrm{C}$ comercial a $1600 \mathrm{rpm}$ a un intervalo de potencial de 0.2 a $0.8 \mathrm{~V} / \mathrm{RHE}$. En estas VLs se observan las curvas de corriente-potencial (I-E) obtenidas por la técnica de electrodo de disco rotatorio (EDR) para cada uno de los electrocatalizadores. En las curvas de polarización se presentan las zonas de control cinético de 0.8 a $0.65 \mathrm{~V}$ para $\mathrm{Ru}_{\mathrm{X}} \mathrm{W}_{\mathrm{Y}} \mathrm{S}_{\mathrm{Z}} / \mathrm{C}$, de 0.8 a $0.5 \mathrm{~V}$ para el $\mathrm{Ru}_{\mathrm{X}} \mathrm{W}_{\mathrm{Y}} \mathrm{S}_{2 Z} / \mathrm{C}$ y $\mathrm{Ru}_{\mathrm{X}} \mathrm{W}_{\mathrm{Y}} \mathrm{S}_{2 Z} / \mathrm{NTC}$ y de 0.8 a $0.75 \mathrm{~V}$ para el $\mathrm{Pt} / \mathrm{C}$; la zona de control mixto está en los intervalos: $0.65-0.2 \mathrm{~V}$ para el $\mathrm{Ru}_{\mathrm{X}} \mathrm{W}_{\mathrm{Y}} \mathrm{S}_{2 \mathrm{Z}} / \mathrm{C}, 0.5-0.2$ $\mathrm{V}$ para el $\mathrm{Ru}_{\mathrm{X}} \mathrm{W}_{\mathrm{Y}} \mathrm{S}_{2 Z} / \mathrm{C}$ y $\mathrm{Ru}_{\mathrm{X}} \mathrm{W}_{\mathrm{Y}} \mathrm{S}_{2 \mathrm{Z}} / \mathrm{NTC}$ El comportamiento para la RRO se lleva a cabo mediante un control mixto de transferencia electrónica y transporte de masa en todos los electrocatalizadores, sin embargo, la transferencia de electrones es lenta en relación al transporte de masa y esta etapa lenta es la determinante del proceso global.

El análisis comparativo de las curvas de polarización en los electrocatalizadores sintetizados $\mathrm{Ru}_{\mathrm{X}} \mathrm{W}_{\mathrm{Y}} \mathrm{S}_{\mathrm{Z}} / \mathrm{C} \mathrm{Ru}_{\mathrm{X}} \mathrm{W}_{\mathrm{Y}} \mathrm{S}_{2 Z} / \mathrm{C}$ y $\mathrm{Ru}_{\mathrm{X}} \mathrm{W}_{\mathrm{Y}} \mathrm{S}_{2 Z} / \mathrm{NTC}$ con respecto al $\mathrm{Pt} / \mathrm{C}$, que es el electrocatalizador de referencia, indica que el material $\mathrm{Ru}_{\mathrm{X}} \mathrm{W}_{\mathrm{Y}} \mathrm{S}_{\mathrm{Z}} / \mathrm{C}$ es similar al Pt/C. El electrocatalizador $\mathrm{Ru}_{\mathrm{X}} \mathrm{W}_{\mathrm{Y}} \mathrm{S}_{\mathrm{Z}} / \mathrm{C}$ inicia la RRO a potenciales más positivos que los electrocatalizadores sintetizados, además este material genera la mayor cantidad de corriente (A) de los materiales de rutenio, aunque el valor es menor que el $\mathrm{Pt} / \mathrm{C}$. Se observa un desplazamiento (E/V vs RHE) en la zona de transferencia electrónica y el transporte de masa (zona mixta), respecto al electrocatalizador de referencia con un intervalo mayor a $0.1 \quad \mathrm{~V}$. La respuesta electrocatalítica de los materiales sintetizados con una relación molar 1:2 es similar, la cinética en ambos electrocatalizadores es lenta; esto confirma los resultados de caracterización fisicoquímica discutidos anteriormente, referente a la formación de aglomerados y el aumento en el tamaño de partículas, por lo que disminuye la actividad catalítica para la RRO. De acuerdo con lo anterior, se sugiere que el electrocatalizador $\mathrm{Ru}_{\mathrm{X}} \mathrm{W}_{\mathrm{Y}} \mathrm{S}_{\mathrm{Z}} / \mathrm{C}$ presenta una mejor actividad, por lo tanto, la reacción química con una relación molar $1: 1$ es conveniente debido a que se obtienen partículas de tamaño nanométrico, lo cual también contribuye en este resultado para lograr una mejor actividad hacia la RRO.

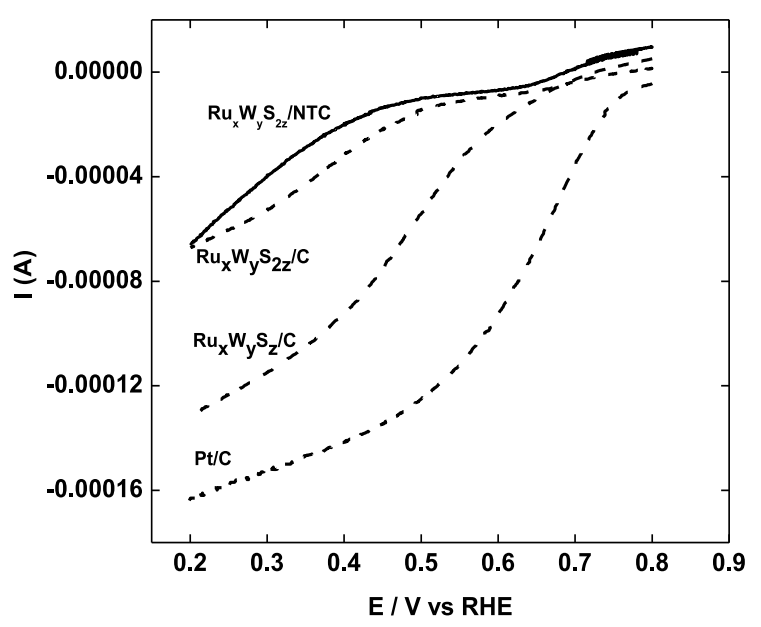

Figura 6. Voltamperogramas lineales. de $\mathrm{Ru}_{\mathrm{X}} \mathrm{W}_{\mathrm{Y}} \mathrm{S}_{Z} / \mathrm{C}$ $\mathrm{Ru}_{\mathrm{X}} \mathrm{W}_{\mathrm{Y}} \mathrm{S}_{2 Z} / \mathrm{C}$ y $\mathrm{Ru}_{\mathrm{X}} \mathrm{W}_{\mathrm{Y}} \mathrm{S}_{2 Z} / \mathrm{NTC}$.

En la Tabla 1 se presentan los resultados obtenidos de la densidad de corriente generada, la eficiencia de los electrocatalizadores $\mathrm{Ru}_{\mathrm{X}} \mathrm{W}_{\mathrm{Y}} \mathrm{S}_{\mathrm{Z}} / \mathrm{C}$, $\mathrm{Ru}_{\mathrm{X}} \mathrm{W}_{\mathrm{Y}} \mathrm{S}_{2 Z} / \mathrm{C}$ y $\mathrm{Ru}_{\mathrm{X}} \mathrm{W}_{\mathrm{Y}} \mathrm{S}_{2 Z} / \mathrm{NTC}$ con respecto al $\mathrm{Pt} / \mathrm{C}$ y la eficiencia entre los electrocatalizadores sintetizados. Estos resultados indican que el $\mathrm{Ru}_{\mathrm{X}} \mathrm{W}_{\mathrm{Y}} \mathrm{S}_{\mathrm{Z}} / \mathrm{C}$ es un $38 \%$ más eficiente para la generación de corriente eléctrica que el $\mathrm{Ru}_{\mathrm{X}} \mathrm{W}_{\mathrm{Y}} \mathrm{S}_{2 Z} / \mathrm{C}$ y $\mathrm{Ru}_{\mathrm{X}} \mathrm{W}_{\mathrm{Y}} \mathrm{S}_{2 Z} / \mathrm{NTC}$ al actuar como electrodo catódico en medio acido, los valores muestran que la corriente que genera el electrocatalizador $\mathrm{Ru}_{\mathrm{X}} \mathrm{W}_{\mathrm{Y}} \mathrm{S}_{\mathrm{Z}} / \mathrm{C}$ se aproxima a la corriente del $\mathrm{Pt} / \mathrm{C}$. El estudio comparativo de los electrocatalizadores analizados en este trabajo sugiere que la cantidad de $\mathrm{Pt} / \mathrm{C}$ puede ser optimizada a través del método de síntesis. En este contexto, el uso de calcogenuros contribuye a disminuir la cantidad de fase metálica activa, además el rol que desempeñan el tungsteno y el azufre como promotores catalíticos también puede contribuir a mejorar la actividad y estabilidad de los electrocatalizadores basados en nanopartículas homogéneas y altamente dispersas [14, 15].

\section{Conclusiones}

En este trabajo se logró la síntesis de nanopartículas de $\mathrm{Ru}-\mathrm{WS}_{2}$ mediante un método sencillo por reacciones químicas y un tratamiento térmico a baja temperatura. El material obtenido con una relación molar 1:1 es más homogéneo y presenta un comportamiento electrocatalítico mejor para la RRO que el material con una relación molar 1:2. Este resultado se atribuye a la formación de aglomerados y a la cantidad en exceso de tiosales $\left(\mathrm{WS}_{2}\right)$ que inhiben los sitios activos del electrocatalizador para la reacción en estudio, por lo tanto su eficiencia disminuye. 
Tabla 1. Electrocatalizadores estudiados para la RRO.

\begin{tabular}{cccc}
\hline Material & $\begin{array}{c}\text { Densidad de corriente } \\
(\mathbf{m A})\end{array}$ & $\begin{array}{c}\text { Eficiencia con respecto } \\
\text { al Pt/C (\%) }\end{array}$ & $\begin{array}{c}\text { Eficiencia entre los } \\
\text { electrocatalizadores sintetizados }\end{array}$ \\
\hline $\mathrm{Pt} / \mathrm{C} \mathrm{Comercial}$ & 0.18 & $100 \%$ & $100 \%$ \\
$\mathrm{Ru}_{\mathrm{X}} \mathrm{W}_{\mathrm{Y}} \mathrm{S}_{\mathrm{Z}} / \mathrm{C}$ & 0.13 & $81 \%$ & $62 \%$ \\
$\mathrm{Ru}_{\mathrm{X}} \mathrm{W}_{\mathrm{Y}} \mathrm{S}_{2 \mathrm{Z}} / \mathrm{C}$ & 0.07 & $43 \%$ & $62 \%$ \\
$\mathrm{Ru}_{\mathrm{X}} \mathrm{W}_{\mathrm{Y}} \mathrm{S}_{2 \mathrm{Z}} / \mathrm{NTC}$ & 0.07 & $43 \%$ & \\
\hline
\end{tabular}

La síntesis de electrocatalizadores eficientes como el $\mathrm{Pt} / \mathrm{C}$ puede ser optimizada, mediante el uso de calcogenuros, ya que estos materiales estabilizan los centros catalíticamente activos si se utilizan en una relación molar adecuada que permita obtener un tamaño de partículas a escala nanométrica y con alta dispersión. En este trabajo se obtuvieron nanopartículas de rutenio de $2 \mathrm{~nm}$.

Los materiales sintetizados pueden ser propuestos como cátodos en medio ácido para la RRO. Sin embargo, es necesario realizar más estudios fisicoquímicos para determinar la naturaleza de la especie activa y la interacción de los elementos que favorecen a la RRO, algunos estudios complementarios están en proceso por los autores.

\section{Agradecimientos}

Los autores agradecen a la DGEST y al CONACYT por el apoyo de las becas de Doctorado, así como el soporte financiero del proyecto CB-133714 e igualmente agradecen el apoyo del CIE-UNAM y al CIMAV, S. C., por la estancia para realizar las caracterizaciones.

\section{Referencias}

1. Williams, M. C.; Fuel Cell Handbook, U.S. Department of Energy; Washington, 2000, 5th ed., 1-37.

2. Nagappan, R.; Sanjeev, M.; Adv. in Phys. Chem. 2011, 117.

3. Masahirom, W.; Haruhiko, S.; Paul, S. J. Electroanal. Chem. 1989, 261, 375-387.

4. Jeffrey, A. P.; Glen, E. S., J. Electrochem. Soc. 1994, 141, 425-430.

5. Alonso-Núñez G.; Yang, J.; Siadati, M. H.; Chianelli, R. R., Inorg. Chem. Acta 2001, 325, 193-197.

6. Rusell, R. Chianelli, Mohammad, H. Siadati, Myriam Perez de la Rosa, Catal. Rev. 2006, 48, 1-41.

7. Dianxue, C.; Andrzej, W.; Junji, I.; Alonso-Vante, N., J. of The Electrochemical Soc. 2006, 153, A869-874.

8. Alonso-Núñez G.; Gochi-Ponce Y.; Barbosa, R.; Arriaga, L. G.; Alonso-Vante N.; J. Electrochem. Soc. 2006, 3, 189197.

9. Huirache-Acuña, R.; Albiter, M. A.; Paraguay-Delgado, F.;. Alonso-Núñez, G. Foro de Ing. e Inv. en Mat. 2007, 4, 4045.
10. Gochi-Ponce, Y.; Síntesis y caracterización de nanoestructuras a base de calcogenuros de metales de transición $\mathrm{M}\left(\mathrm{MS}_{2}\right)_{\mathrm{x}}$ como electrocatalizadores en la celda de combustible de membrana polimérica, Tesis de doctorado, CIMAV, Chihuahua, México Julio de 2007.

11. Gasser, R.P.H. An Introduction to Chemisorption and Catalysis by Metals, Oxford Science Publications, New York, 1985.

12. Kinoshita, K.; Ross, P. N. J. Electroanal. Chem. and Interfacial Electrochem. 1977, 48, 157-166.

13. Pinheiro, A. L. N.; Zei, M. S.; G. Ertl, Phys. Chem. Chem. Phys. 2005, 7, 1300-1309.

14. Yongjun, Feng.; Gago, A.; Timperman, L.; Vante, N. A. Chalcogenide metal centers for oxygen reduction reaction: Activity and tolerance, Electrochim. Acta, 2011, 56, 10091022.

15. Ermete, A.; Gonzalez, E. R. Tungsten-based materials for fuel cell applications, App. Catal. B: Env. 2010, 96, 245266. 